\title{
The Effects of Mindfulness on Self-Rumination, Self-Reflection, and Depressive Symptoms: A Research Proposal
}

\author{
Mabel Yu \\ Department of Psychology, Mount Royal University \\ 4825 Mt Royal Gate, Calgary, AB, T3E 6K6 \\ m.mabelyu@gmail.com
}

Review Editors

Bruce Hoffman ${ }^{1}$, Hanaa Elmoghrabi ${ }^{2}$, Alain Morin ${ }^{3}$

Editor

Famira Racy 4

\begin{abstract}
The investigation of mindfulness has increased significantly over the past decade regarding its efficacy as a clinical tool, particularly in the treatment of depression. Mindfulness is often conceptualized as a mental state characterized by present-moment, non-judgmental attention and awareness. Past researchers have suggested that mindfulness is linked to reduction of self-rumination (i.e. maladaptive self-focused attention to one's self-worth) through promotion of concrete focus and inhibition of automatic elaboration of intrusive thoughts. Moreover, mindfulness also promotes low-level construal thinking (i.e. concrete thinking) which competes against high-level construal thinking (i.e. abstract thinking). Researchers have proposed that self-rumination involves high-level construal of the self and others, which could increase the likelihood of experiencing negative moods. On the other hand, mindfulness may potentially promote self-reflection (i.e. adaptive self-focused attention to the self) while inhibiting selfrumination. The purpose of this paper is to propose a research idea that will explore the relationship between mindfulness, self-rumination, self-reflection, and depressive symptoms (i.e., low mood, anhedonia or ability to feel pleasure, and changes in sleep). The findings of the proposed research may have significant implications for treatment of depressive symptoms and for promotion of positive outcomes such as mitigation of self-rumination and enhancement of self-reflective processes through potential effects of mindfulness.
\end{abstract}

Keywords: mindfulness $\cdot$ self-rumination $\cdot$ self-reflection $\cdot$ depression $\cdot$ MBSR

1 BA (Hns.) Psychology · York University

2 BA · Mount Royal University

${ }^{3} \mathrm{PhD} \cdot$ Department of Psychology $\cdot$ Mount Royal University

${ }^{4}$ Adler University (MA candidate) · Mount Royal University Psychology Alumna 


\section{Mindfulness \& Depression}

The impressive surge in the popularity of mindfulness in psychology has generated significant research over the past decades (e.g., Baer et al., 2008; Bishop et al., 2004; Brown \& Falkenström, 2010; Kabat-Zinn, 1994; Ryan, 2003). Although mindfulness has its roots in religion and philosophies, its application in contemporary psychology is nondenominational in nature. Mindfulness is often conceptualized in contemporary psychology as a mental state characterized by present-moment awareness and attention to one's inner experiences, such as thoughts, feelings, emotions, and sensations (Kabat-Zinn, 1994). These experiences are observed, attended to, and accepted by the self with a non-judgmental attitude (Bishop et al., 2004).

Although mindfulness can be understood as a dispositional trait that varies from one person to another, one's mindfulness level can be increased through mindfulness-based training and practices (i.e., meditation; Baer et al., 2008; Brown \& Ryan, 2003; Falkenström, 2010). Recent shifts in the literature shows researchers investigating the effectiveness of mindfulness as a clinical tool to treat a wide range of psychological disorders such as depression and anxiety. Moreover, results support the effectiveness of mindfulness-based interventions for a number of clinical and nonclinical levels of problems, as well as the improvement of overall psychological functioning (e.g., Baer et al., 2008; Grossman et al., 2004).

As popularized by Mindfulness-Based Cognitive Therapy (MBCT; Segal, Williams, \& Teasdale, 2002), which was developed as treatment to prevent relapse in recurrent major depression, a wealth of empirical research has suggested a negative relationship between mindfulness and depressive symptoms (e.g., affective or emotional symptoms of depression and anxiety as well as dysfunctional attitudes and rumination) such that as mindfulness increases through intervention, depressive symptoms decrease, contributing to prevention of depression relapse (Ramel, Goldin, Carmona, \& McQuaid, 2004).

\section{Mindfulness \& Self-Rumination}

Mindfulness researchers have postulated that the efficacy of mindfulness training can be attributed to reduction in maladaptive self-rumination (e.g., Heeren \& Philippot, 2011; Kingston et al., 2007;
Ramel, Goldin, Carmona, \& McQuaid, 2004). According to Morin (2011), self-rumination is the anxious attentional focus one pays to one's own selfworth. Researchers have found that self-rumination is linked to unhealthy psychological functioning such as a number of clinical syndromes including anxiety and depression (Joireman, 2004; Joireman, Parrott, \& Hammersla, 2002). Although the exact mechanisms and causal directions behind these relationships are unclear, researchers have suggested that mindfulness training emphasizes a specific type of attentional focus, specifically concrete focus (e.g., focusing on one's breathing). Concrete focus prevents the elaboration of intrusive thoughts, which is a seemingly automatic process (Segal et al., 2002). For example, focusing on one's breathing narrows one's access to the cognitive resources needed to carry out ruminative processes. Therefore, mindfulness promotes concrete focus which inhibits secondary processing of thoughts, feelings, and sensations that predominantly occur during the process of self-rumination (Bishop et al., 2004; Heeren \& Philippot, 2011).

Researchers have further proposed that selfrumination involves high-level construal about the self and mood. High-level construal consists of general and abstract mental representations whereas low-level construal consists of the specific, concrete representations of experiences (Watkins, 2008). While low-level construal is typically associated with adaptive thinking, high-level construal is known to increase vulnerability towards developing an emotional disorder (Gibbs \& Rude, 2004; van Minnen et al., 2005). This is because high-level construal reduces concreteness of thoughts, which tends to lead to a negative overgeneralization of events (e.g., 'I always disappoint people', Heeren \& Philippot, 2011). A study by Watkins et al. (2008) showed that participants who were trained to adopt a low-level processing mode exhibited less emotional reactivity after failing a stressful task than those who were trained to process with highlevel construal. Further, researchers have found that high-level construal, which is associated with self-rumination, is also linked to depression relapse and can intensify depressive symptoms (Michalak, Hölz, \& Teismann, 2011; Ramel, Goldin, Carmona, \& McQuaid, 2004).

Maladaptive high-level construal can contribute to negative psychological outcomes but past researchers have suggested that mindfulness could act as a safeguard against these outcomes. The notion that mindfulness is linked to reduction of self- 
rumination and increase of self-reflection through related processes is further supported by empirical evidence showing that mindfulness prevents the processing of high-level construal by promoting low-level construal processing. In essence, mindfulness reduces ruminative thinking by transforming automatic, rather maladaptive, cognitive processes that lend to abstract thinking and self-rumination to more adaptive and purposeful cognitive processes that lend to concrete thinking and enhancement of specific details indicative of self-reflection. For example, a study by Heeren, Broeck, and Philippot (2009) showed that mindfulness training led to participants recalling a greater number of specific details of an emotional event as well as a reduction in the number of generic and abstract accounts of emotional events.

\section{Self-Rumination \& Self-Reflection}

Researchers on self-rumination have often juxtaposed this concept with self-reflection. Trapnell and Campbell (1999) dichotomized self-rumination and self-reflection as two subtypes of self-focused attention, with the latter being the adaptive type. Self-reflection, as defined by Morin (2011), is the "genuine curiosity about the self, where the person is intrigued and interested in learning more about his or her emotions, values, thought processes, attitudes, etc." (p. 809). Unlike self-rumination, self-reflection is linked to a number of positive outcomes such as, increased self-knowledge (Şimşek, Ceylandag, \& Akcan, 2013), subjective happiness (Elliott \& Coker, 2008), and self-regulation (Thomsen, Tønnesvang, Schnieber, \& Olesen, 2011). Alternatively, previous research has indicated that self-rumination is linked to decreased subjective happiness (Elliott \& Coker, 2008), impaired interpersonal skills (Takano, Sakamoto, \& Tanno, 2011), and can exacerbate depressive symptoms (Takano \& Tanno, 2009).

The relationship between self-rumination and self-reflection can be understood through the concept of decentering. Emerged from mindfulness theory, decentering is the objective processing of one's internal experiences (e.g., feelings, thoughts, and sensations) through the distancing of oneself from these experiences (Safran \& Segal, 1990). Since the key idea of decentering is the changing of one's relationship with the contents of their thoughts and emotions, researchers have suggested that decentering mitigates rumination because decentered individuals can disengage once becoming aware of their ruminative thoughts (Teasdale et al., 2002). Consistent with this theory, past findings have shown a negative association between decentering and rumination, and decentering has been found to be negatively associated with depression (Fresco et al., 2007; McCracken, Barker \& Chilcot, 2014; Mori \& Tanno, 2015).

In contrast to self-rumination, self-reflection is considered to be positively associated with decentering (Lau et al., 2006). In a study by Mori and Tanno (2015), individuals with a higher degree of reported self-reflection revealed more decentering and less depressive symptoms. These findings lend support to the proposed hypothesis that through self-reflection, individuals can attend objectively to their inner experiences-both adaptive and maladaptive-which may allow them to more effectively regulate their thoughts and feelings.

Since self-rumination and self-reflection are often contrasted against each other, following the notion that mindfulness reduces self-rumination, then, mindfulness should essentially promote selfreflection. Although the conceptualization of selfreflection is not entirely congruent with the definition of mindfulness, some aspects of the construct still encompass several mindfulness dimensions, such as a sense of curiosity and openness (Baer et al., 2008). Results from a study by Heeren and Philippot (2011) supports this claim with findings that participants who underwent mindfulness training reported decreases in rumination and increases in adaptive rumination-which can be seen as self-reflection.

\section{Suggestions for Future Research}

To my knowledge, there is no research investigating the links between mindfulness, depressive symptoms, self-rumination, and self-reflection while investigating the effects of mindfulness on these variables. It is crucial to address such questions raised in this research proposal in order to utilize mindfulness in ways that will lend to significant positive effects. Thus, in the following sections I propose a research idea that potentially addresses the effects of mindfulness on depressive symptoms, self-rumination, and self-reflection. Specifically, the research will attempt to answer the questions of whether the cultivation of mindfulness significantly reduces both self-rumination and depressive symptoms while increasing self-reflection in a non-clinical sample. In terms of the research hypotheses, in 
the mindfulness treatment group there should hypothetically be (1) a significant increase in mindfulness, (2) a significant increase in self-reflection, (3) a significant decrease in self-rumination, and (4) a significant decrease in depressive symptoms as compared to the waitlist control group. The proposed research follows a between-subject (mindfulness treatment group vs. waitlist control) and a within-subject (pre-treatment vs. post-treatment) design.

\section{Proposed Methods}

Materials \& Procedure. After recruitment, the participants will be asked to complete a demographics form that collects basic information such as age, gender and ethnicity. On the demographics form, participants indicate their experience and/or knowledge regarding mindfulness. Participants are ineligible to participate if they have experience with MBSR or similar types of mindfulness practice. As a repeated-measures design study, participants will be asked to complete a battery of self-report questionnaires twice over the study period. The questionnaires will be counterbalanced to control for order effects, and have been reported as generally valid and reliable measures. This process determines the baseline (pre-test) scores and difference (post-test) scores of mindfulness, self-reflection, rumination, and depressive symptoms.

Mindfulness will be measured using the Mindfulness Attention Awareness Scale (MAAS; Brown \& Ryan, 2003) and depressive symptoms will be measured using the Beck Depression Inventory-II (BDI-II; Beck, Steer, \& Brown, 1996). Self-rumination and self-reflection will be assessed using two measures: the Rumination and Reflection Questionnaire (RRQ; Trapnell \& Campbell, 1999) and the short form of the Cambridge Exeter Repetitive Thought Scale (Mini-CERTS; Barnard, Watkins, Mackintosh, \& Nimmo-Smith, 2007). After filling out the pre-test questionnaires, participants will be randomly assigned to one of two conditions-the mindfulness treatment condition or the waitlist control condition. The participants assigned to the treatment condition will enroll in the MBSR program, whereas the participants in the control condition will be asked to wait 8 weeks until the commencement of the intervention.

Following the 8-week MBSR program or waitlist period and after the questionnaire battery is administered to all participants for a second time, all participants will be debriefed and compensated for their participation. It is worth noting that participants in the control condition should be informed during debriefing that they were assigned to the waitlist condition and that no mindfulness intervention will take place. However, resources such as titles of journal articles and self-help books pertinent to the topic of MBSR and mindfulness will be provided to participants interested in learning more about this topic.

\section{Measures}

RUMINATION \& REFLECTION QUESTIONNAIRE (RRQ). The RRQ (Trapnell \& Campbell, 1999) is a 24-item self-report questionnaire assessing both self-rumination and self-reflection, with 12 items corresponding to each subscale. Participants rate the degree to which they engage in self-reflective and selfruminative thoughts on a 5-point Likert scale $11=$ strongly disagree; 5 = strongly agree). Higher subscale scores indicate higher degrees of self-rumination or self-reflection. An example of a question pertaining to the self-rumination subscale is: "I always seem to be rehashing in my mind recent things I've said or done." An example of a self-reflection item is: "I love exploring my 'inner self". The RRQ has been reported to have acceptable discriminant and convergent validity, as well as acceptable reliability (.90) for both subscales. Trapnell and Campbell (1999) found that the two subscales adequately measure two distinct and independent constructs.

CAMBRIDGE EXETER RePETITIVE THOUght SCALE (SHORTENED). The Mini-CERTS (Barnard et al., 2007) contains 15 items which assess two distinct, negatively correlated dimensions or subscales of rumination: abstract-analytic (AA) rumination which is considered maladaptive ( 7 items), and the other concrete-experiential (CE) which reflects adaptive rumination ( 8 items). Participants are asked to complete the sentence, "When thoughts, feelings, situations or events about me come to mind..." using phrases provided. An example of a phrase pertaining to the CE subscale is: "I seem to be engaged and directly in touch with what is going on around me", whereas an example of an AA phrase is: "I focus on the causes and meanings of what happened." Each item is rated on a 4-point Likert scale (1 = almost never; 4 = almost always) and the scores within each subscale are summed to generate an overall score for that subscale. Past research has reported acceptable reliability (e.g., estimated internal consistency across the items; 
Cronbach's alpha levels of .77 and .80 for the CE and AA subscales respectively; Douilliez et al., 2014).

Mindfulness ATtention Awareness SCALE (MAAS). The MAAS (Brown \& Ryan, 2003) is a 15item self-report measure producing a single-factor of dispositional mindfulness. The MAAS, which is rated on a 6-point Likert scale $(1=$ almost never; 6 = almost always), assesses present-moment awareness and attention of events and experiences. The items include statements like: "I find it difficult to stay focused on what's happening in the present" and "I find myself doing things without paying attention". A sum score is calculated for each participant, with higher scores indicating higher degrees of dispositional mindfulness associated with wellbeing. The MAAS has shown strong convergent and discriminant validity in past research (Brown \& Ryan, 2003).

BECK DEPRESSION INVENTORY-II (BDI-II). The BDI-II (Beck, Steer, \& Brown, 1996) is a 21-item self-report inventory that assesses the severity of depressive symptoms (i.e., low mood, anhedonia or ability to feel pleasure, and changes in sleep) over the past two weeks. Each item is rated on a scale value of 0 to 3 with higher scores reflecting higher degrees of depressive symptoms. The BDI-II is one of the most commonly used instruments for measuring depression with extensive data available on the validity and reliability of the measure (see Beck, Steer, \& Brown, 1996).

InTERVENTION OF Mindfulness-BASEd STRESS REDUCTION (MBSR). Participants in the intervention condition will be asked to partake in the mindfulness-based stress reduction (MBSR) program developed by Kabat-Zinn (1982). The intervention consists of 90-minute group classes that will be held once a week for eight weeks. Formal mindfulness practices are taught in the forms of breathing exercises, body scan (mindfully acknowledging the sensations that arise in the body), hatha yoga (practice of postures and poses), as well as walking and sitting meditation. Additionally, participants will be asked to engage in 45 minutes of meditation six times a week and to document a homework log each time. Participants will be presented with a guided meditation audio to assist them during their meditation sessions.

\section{Hypothetical Results}

Data analysis of pre-test (baseline) scores of the two conditions should hypothetically reveal nonsignificant differences between the mindfulness group and the waitlist group on all of the measures, indicating scores were taken from two similar groups. However, I expect that the pre-test and post-test scores on the MAAS, the RRQ, the MiniCERTS, and the BDI-II for the mindfulness group will change significantly after the treatment intervention in comparison to the waitlist control group. Specifically, the scores between the mindfulness group and the waitlist control group should be hypothetically significantly different at post-test, such that as consistent with the hypotheses, the results should hypothetically show significant decreases in self-rumination and depressive symptoms, as well as significant increases in mindfulness and self-reflection in the mindfulness group as compared to the control group.

\section{Discussion}

The proposed research aims to investigate whether mindfulness intervention, in particular MBSR, would increase adaptive self-reflection, reduce the tendency to self-ruminate, and mitigate depressive symptoms. If the results support the hypotheses, it could suggest that MBSR training could increase mindfulness, increase adaptive processes and decrease maladaptive processes. Furthermore, if the results show that the increase in mindfulness is associated with decreases in self-rumination and increases in self-reflection, it would add support to previous findings that mindfulness does promotes concrete focus and inhibits the abstract elaboration of thoughts (a key component of self-rumination is abstract elaboration of thoughts; Segal et al., 2002). Through the practice of mindfulness skills, such as practicing a non-elaborative and non-judgmental attitude of one's thoughts and feelings, one is able to bring conscious awareness to the present and to act as a curious observer of one's own experiences-which is consistent with the notion of selfreflection (e.g., Morin, 2011).

If the results support significant reduction of depressive symptoms in the mindfulness condition, the findings would be consistent with the myriad of literature that advocates mindfulness as an effective technique in the treatment of depression and related symptoms (e.g., Ramel, Goldin, Carmona, \& McQuaid, 2004). Given the expected results, I postulate that mindfulness works to interrupt the automatic maladaptive cognitive processes that spiral once a negative thought has sprung into the mind of a depressive individual. The content of self-rumina- 
tion (repetitive thoughts, emotions, feelings) is similar to the content involved in mindfulness; however mindfulness encourages individuals to notice their thoughts, emotions, or feelings. Contrary to self-rumination, mindfulness promotes one's own non-judgment and non-reactivity to these processes, observing them as merely temporary experiences rather than reflections of oneself (KabatZinn, 1994). By doing so, mindfulness in turn enables individuals to stand as objective observers and to identify solutions to their current problems by uncovering their repertoire of available resources. On the contrary, if one is not mindful and engages in ruminative thinking, as a result, this may restrict one's ability to identify possible solutions. Moreover, one may be more susceptible to losing oneself to the vicious cycle of continuous negative thinking when ruminating rather than when using mindfulness.

Recent shifts in psychotherapy have included mindfulness in a number of clinical approaches, as research findings continue to indicate support for its effectiveness in promoting positive outcomes. A strength of this proposed study is that it extends beyond previous research by investigating both selfrumination and self-reflection in the context of mindfulness. To my knowledge, the links between these constructs have not been extensively explored; thus, research on these relationships is strongly needed. Furthermore, the findings from the present research have substantial treatment implications for depression, and continue to support the efficacy of mindfulness in promoting positive outcomes such as in reducing self-rumination and increasing self-reflection tendencies. One limitation is the use of self-report measures in that the participant responses are vulnerable to social desirability bias. For example, participants in the mindfulness group may intentionally or unintentionally polarize their responses to reflect a more favorable score in the post-test. Another limitation of the study is the time commitment required of participants in the study. A significant amount of participant attrition would certainly undermine the data and the quality of the study.

Lastly, the direction of this field of research should expand on the current study in the investigation of self-rumination and self-reflection by using behavioural measures to examine these two constructs in addition to self-report measures. Moreover, research should focus on disentangling the possible links between mindfulness and such variables by looking at whether there are other factors that influence the intensity of the effects of mindfulness on self-rumination and self-reflection. Finally, an idea for future research could be to investigate whether decreases in self-rumination as well as increases in self-reflection tendencies mediate the relationship between mindfulness and depressive symptoms.

\section{References}

Baer, R. A., Smith, G., Lykins, E. L. B., Button, D., Krietemeyer, J., Sauer, S., Walsh, E., Duggan, D. \& Williams, J.G. (2008). Construct validity of the five facets of mindfulness questionnaire in meditating and non-meditating samples. Assessment, 15(3), 329-342.

Barnard, P., Watkins, E., Mackintosh, B. \& Nimmo Smith, I. (2007). Getting stuck in a mental rut: Some process and experiential attributes. Paper presented at the 35th congress of the British Association for Behavioural and Cognitive Psychotherapies, Brighton, England.

Beck, A. T., Steer, R. A., \& Brown, G. K. (1996). Manual for the BDI-II. San Antonio, TX: Psychological Corporation.

Bishop, S. R., Lau, M., Shapiro, S., Carlson, L., Anderson, N. D., Carmody, J. Segal, Z., Abbey, S., Speca, M., Velting, D. \& Devins, G. (2004). Mindfulness: A proposed operational definition. Clinical Psychology: Science and Practice, 11, 230241.

Brown, K. W., \& Ryan, R. M. (2003). The benefits of being present: Mindfulness and its role in Psychological well-being. Journal of Personality and Social Psychology, 84, 822-848.

Douilliez, C., Heeren, A., Lefèvre, N., Watkins, E., Barnard, P., \& Philippot P. (2014). Validation de la version française d'un questionnaire évaluant les pensées répétitives constructives et non constructives. Canadian Journal of Behavioral Science, 46(2), 185-192.

Elliott, I., \& Coker, S. (2008). Independent self-construal, self-reflection, and self-rumination: A path model for predicting happiness. Australian Journal of Psychology, 60, 127-134.

Falkenström, F. (2010). Studying mindfulness in experienced meditators: A quasi-experimental approach. Personality and Individual Differences, 48, 305-310.

Fresco, D. M., Moore, M. T., van Dulmen, M. H. M., Segal, Z. V., Ma, S. H., Teasdale, J. D., \& Williams, J. M. G. (2007). Initial psychometric properties of the experiences questionnaire: Validation of 
self-report measure of decentering. Behavior Therapy, 38, 234-246.

Gibbs, B. R., \& Rude, S. S. (2004). Overgeneral autobiographical memory as depression vulnerability. Cognitive Therapy and Research, 28, 511526.

Grossman, P., Niemann, L., Schmidt, S., \& Walach, H. (2004). Mindfulness-based stress reduction and health benefits: A meta-analysis. Journal of Psychosomatic Research, 57(1), 35-43.

Heeren, A., \& Philippot, P. (2011). Changes in ruminative thinking mediate the clinical benefits of mindfulness: Preliminary findings. Mindfulness, 2, 8-13.

Heeren, A., Van Broeck, N., \& Philippot, P. (2009). The effects of mindfulness on executive processes and autobiographical memory specificity. Behaviour Research and Therapy, 47, 403-409.

Joireman, J. A. (2004). Empathy and the self-absorption paradox II: Self-rumination and self-reflection as mediators between Shame, Guilt, and Empathy. Selfand Identity, 3(3), 225-238.

Joireman, J. A., Parrott, L., \& Hammersla, J. (2002). Empathy and the self-absorption paradox: Support for the distinction between self-rumination and self-reflection. Self and Identity, 1, 53-65.

Kabat-Zinn, J. (1994). Wherever you go, there you are: Mindfulness meditation in everyday life. New York, NY: Hyperion.

Kingston, T., Dooley, B., Bates, A., Lawlor, E., \& Malone, K. (2007). Mindfulness-based cognitive therapy for residual depressive symptoms. Psychology and Psychotherapy-Theory, Research and Practice, 80, 193-203.

Lau, M. A., Bishop, S. R., Segal, Z. V., Buis, T., Anderson, N. D., Carlson, L., \& Devins, G. (2006). The Toronto mindfulness scale: Development and validation. Journal of Clinical Psychology, 62, 1445-1467.

McCracken, L. M., Barker, E., \& Chilcot, J. (2014). Decentering, rumination, cognitive defusion, and psychological flexibility in people with chronic pain. Journal of Behavioural Medicine, 37, 1215-1225.

Mori, M., \& Tanno, Y. (2015). Mediating role of decentering in the associations between self-reflection, self-rumination, and depressive symptoms. Psychology, 6, 613-621.

Morin, A. (2011) Self-awareness part 1: Definition, measures, effects, functions, and antecedents. Social and Personality Psychology Compass, 5(10), 807-823.
Ramel, W., Goldin, P. R., Carmona, P. E., \& McQuaid, J. R. (2004). The effects of mindfulness meditation on cognitive processes and affect in patients with past depression. Cognitive Therapy and Research, 28, 433-455.

Safran, J., \& Segal, Z. (1990). Interpersonal process in cognitive therapy. New York, NY: Basic Books.

Segal, Z. V., Williams, J. M. G., \& Teasdale, J. D. (2002) Mindfulness-based cognitive therapy for depression: A new approach to preventing relapse. New York, NY: Guildford Press.

Simsek, O. F., Ceylandag, A. E., \& Akcan, G. (2013). The need for absolute truth and self-rumination as basic suppressors in the relationship between private self-consciousness and mental health. The Journal of General Psychology, 140, 294-310.

Takano, K., \& Tanno, Y. (2009). Self-rumination, selfreflection, and depression: Self-rumination counteracts the adaptive effect of self-reflection. Behavior Research and Therapy, 47, 260264.

Takano, K., Sakamoto, S., \& Tanno, Y. (2011). Ruminative and reflective forms of self-focus: Their relationships with interpersonal skills and emotional reactivity under interpersonal stress. Personality and Individual Differences, 51, 515-520.

Teasdale, J. D., Moore, R. G., Hayhurst, H., Pope, M., Williams, S., \& Segal, Z. V. (2002). Metacognitive awareness and prevention of relapse in depression: Empirical evidence. Journal of Consulting and Clinical Psychology, 70, 275-287.

Thomsem, D. K., Tønnesvang, J., Schnieber, A., \& Olesen, M. H. (2011). Do people ruminate because they haven't digested their goals? The relations of rumination and reflection to goal internalization and ambivalence. Motivation and Emotion, 35, 105-117.

Trapnell, P. D., \& Campbell, J. D. (1999). Private selfconsciousness and the Five-Factor Model of personality: Distinguishing rumination from reflection. Journal of Personality and Social Psychology, 76(2), 284-304.

Minnen, A., Wessel, I., Verhaak, C., \& Smeenk, J. (2005). The relationship between autobiographical memory specificity and depressed mood following a stressful life event: A prospective study. The British Journal of Clinical Psychology, 44, 405-415.

Watkins, E. R. (2008). Constructive and Unconstructive Repetitive Thought. Psychological Bulletin, 134(2), 163-206. 